\title{
Experimental Multi-scale analysis of Carbon/Epoxy Composites Nano-Reinforced by Carbon Nanotubes/Multi-layer Graphene
}

\author{
Suchilla Garcia Leão ${ }^{a}$, Marina Georgia de Melo Martins ${ }^{a}$, Nathalia Caroline Ferreira Menezes ${ }^{a}$, \\ Fernanda Luiza Rinco de Mendonça Lima ${ }^{a}$, Camila Fernanda Silva ${ }^{a}$, Guilherme Costa Arantes ${ }^{a}$,
}

Antonio Ferreira Ávila ${ }^{b, *}$
aPrograma de Pós-Graduação em Engenharia Mecânica, Universidade Federal de Minas Gerais, 6627, Antonio Carlos Avenue, 31270-901, Belo Horizonte, MG, Brazil.
${ }^{b}$ Departamento de Engenharia Mecânica, Universidade Federal de Minas Gerais, 6627, Avenida Antonio Carlos, 31270-901, Belo Horizonte, MG, Brazil.

Received: October 30, 2017; Revised: December 09, 2017; Accepted: December 10, 2017

\begin{abstract}
Nano-size structures, nanotubes/multi-layer graphene (CNT/MLG), were dispersed into epoxy system by a combination of sonication and high shear mixing. To avoid CNT/MLG agglomeration, a non-covalent functionalization were performed using surfactants, CO890 - Polyoxyethylene (40) nonylphenyl ether and SDBS - sodium dodecyl benzene sulfonate. Raman spectroscopy and Fourier transformed Infra-red analysis indicated that CO890-MLG and SDBS-CNT have strong chemical interactions. Atomic force microscopy revealed formation of "spike-like" nanostructures for SDBSCNT combination and "wall-like" nano-shape structures for the CO890-MLG ones. Tensile tests data indicate no significant change on stiffness. In average, the ultimate stress increased around $18 \%$, while toughness had an improvement close to $62 \%$. SDBS-CNT and CO890-MLG at small concentrations $(0.075$ wt. $\%)$ are probably the best options for nano-reinforcement of carbon/epoxy composites.
\end{abstract}

Keywords: Carbo fiber/epoxy laminates, graphene, carbon nanotubes, failure mode, hybrid nanocomposites.

\section{Introduction}

Multi-layer graphene (MLG) and carbon nanotubes (CNT) are two of allotropes structures which present fascinating and unique properties due to the combination of the reduced dimensions and the different lattice structure $^{1}$. As described by Kuilla et al. ${ }^{2}$, the tensile strength of graphene is much higher than steel, aramid fibers or natural rubber; its thermal conductivity is higher than all these materials and the electrical conductivity is also higher than these materials, except for steel. Lee et al. ${ }^{3}$ mentioned that graphene effective elastic modulus follows a normal distribution with a peak close to $1.0 \mathrm{TPa}$ and as commented by Avila et al. ${ }^{4}$, this high stiffness and elevated strength can be attributed to the elevated specific surface area $\left(\approx 2600 \mathrm{~m}^{2} / \mathrm{g}\right)$ and the strong carbon-carbon covalent bonds.

The recent changes on CNT and graphene synthesis led to a decreased into its cost. As a consequence, the number of researchers using carbon based nanostructures increased, and the results on nano-reinforcement of composites laminates are encouraging. Among those researchers are Kim et $\mathrm{al}^{5}$ whom described no significant increase on tensile properties of the addition of CNTs to carbon fibers/epoxy laminates. Nonetheless, they

*e-mail: avila@ufmg.br noticed an enhancement on flexural modulus $(\approx 12 \%)$ and strength $(\approx 18 \%)$ with the addition of $0.3 \mathrm{wt}$. $\%$ of CNT to the epoxy system. These properties enhancement can be attributed to changes into flexural failure mechanisms.

To avoid the graphene/CNT cluster/agglomerates Tkalya et $a l^{6}$ recommended the usage of surfactants. According to them, during the dispersion process by sonication (by bath or horn) carbon based nanostructures are exfoliated into individual nanostructure. This debundle/exfoliation process is due to the mechanical energy provided by sonication which overcomes the Van der Waals interactions between CNTs bundles or graphene platelets. If this energy is removed, the individual nanostructures have the tendency to agglomerate. The usage of surfactants during the sonication promotes the surfactant molecules adsorption onto individual exfoliated nanostructures and consequently avoiding agglomeration. The idea of using surfactants into aqueous solution to disperse carbon based nanostructures and later on incorporate the exfoliated nanostructures into polymeric solution was proposed by $\mathrm{Pu}$ et $\mathrm{al}^{7}$. The called the attention that pristine graphene is naturally hydrophobic, therefore, producing a stable suspension (graphene in water or organic solvent) is a critical issue during the nanocomposite synthesis. Both Tkalya et $\mathrm{al}^{6}$ and $\mathrm{Pu}$ et $\mathrm{al}^{7}$ investigated the usage of 
surfactants as carbon based nanostructures (graphene or CNT) dispersion "facilitator". As discussed by them, from the large number of surfactants two can be considered the most promising, i.e. nonylphenylether (CO890) and sodium dodecyl sulfate (SDS). According to $\mathrm{Mital}^{8}$, the usage of surfactants not only create a stable solution (graphene or CNT in water or organic solvent) but it is also allows the network formation inside the polymer matrix. This percolation process can lead to multifunctional composites, which includes electrical conductivity.

It is clear that CNTs and graphene nanosheets are promising as reinforcement for nanocomposites, but the dispersion process and the cluster formation are problems to be overcome. To incorporate CNTs and graphene into composite materials it is essential to achieve their stable suspension. Due to the van der Waals forces between nanotubes and graphene sheets this structures have the tendency to form clusters/agglomerates. Although covalent chemical functionalization has been proposed to improve the dispersion of these carbon based nanostructures, this method could affect some of their properties. According to Bystrzejewski et al. ${ }^{9}$ as non-covalent modification is based on the adsorption of molecules onto the CNTs/ graphene surface, their structures are not disturbed. To avoid graphene/CNT agglomeration Tkalya et al. ${ }^{6}$ suggested the usage of surfactants. Therefore, a wide variety of surfactants have been studied and used for preparing stable dispersions of CNT/graphene.

This paper investigates how changes on atomic level, non-covalent functionalization, affects the morphology of carbon based nanostructures formation and the overall composite materials mechanical properties. It is important to point-out that this research is focusing on understanding how two different carbon based nanostructures (CNT and MLG) functionalized by surfactants, at optimum or near-optimum conditions ${ }^{6,7}$ can influence the overall composite material mechanical behavior. Therefore, by keeping the carbon/epoxy contents the same and varying the CNT/MLG contents, it will be possible to try to stablish a correlation between these two types of nanostructures and the composites' mechanical response.

\section{Materials and Experimental Procedures}

The carbon nanotubes (CNT) employed in this research were multi-walled carbon nanotubes grown by vapor chemical deposition ${ }^{10}$, with diameters between 5 to $10 \mathrm{~nm}$ and aspect ratio (length/diameter) from 50-100. Graphene was actually a multi-layered graphene (MLG) synthetized by a top-down process which employed graphite as precursor ${ }^{11}$. MLG used in this research had between 5 and 30 layers of mono-layer graphene.

Due to hydrophobic nature of CNT an ionic surfactant (SDBS - sodium dodecyl benzene sulfonate) was used to exfoliate CNT in water ${ }^{8}$. SDBS is supposed to allow $\mathrm{CH}$ of aliphatic groups to interact with carbon nanotubes. For MLG a non-ionic surfactant (IGEPAL CO890 Polyoxyethylene (40) nonylphenyl ether) was used into the exfoliating process in water ${ }^{9}$. It is expected that CO890 will enhance $\mathrm{CH}$ groups in alkanes and $\mathrm{CO}$ groups to interact with graphene layers. As described by Pu et al. ${ }^{7}$, the optimum concentrations for CNT exfoliation in water is $200 \mathrm{ppm}$ of SDBS, while they recommend $300 \mathrm{ppm}$ of CO890 for exfoliation of MLG in water. Non-covalent bonds formed due to surfactant usage were expected to prevent agglomeration of CNT/MLG when dispersed into epoxy systems. Figures $1 \mathrm{~A}-\mathrm{B}$ show the chemical structures for SDBS and CO890, respectively ${ }^{6,7}$.<smiles>CCCCCc1ccc(S(=O)(=O)O[Na])cc1</smiles>

(a)

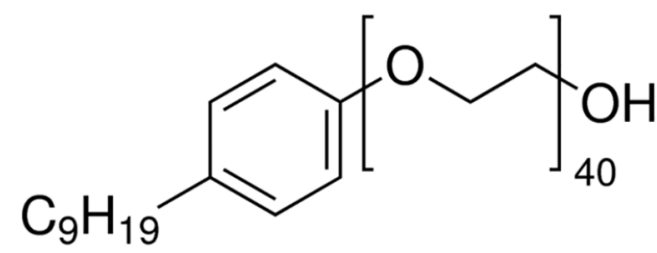

(b)

Figure 1. Surfactants chemical formulas. (a) SDBS; (b) CO890

The epoxy system used is a bisphenol - diglycidyl $A$ resin with an amine hardener. The resin/hardener ratio, in weight, suggested by the manufacturer was 3:1. Table 1 summarizes each material employed and its concentrations. 
Table 1. Materials

\begin{tabular}{ccc}
\hline $\begin{array}{c}\text { Carbon based nanostructures } \\
\text { concentrations [wt.\%] }\end{array}$ & \multicolumn{2}{c}{ Surfactants } \\
\hline Control Samples -0.0 & ---- & ---- \\
CNT -0.075 & 200 & ---- \\
CNT -0.150 & 200 & ---- \\
CNT -0.300 & 200 & ---- \\
MLG -0.075 & --- & 300 \\
MLG -0.150 & ---- & 300 \\
MLG -0.300 & ---- & 300
\end{tabular}

Epoxy system: AR300/AH150**

Carbon fibers: plain weave, $200 \mathrm{~g} / \mathrm{m}^{2 * *}$

Epoxy/carbon fibers ratio: 50/50

*From Sigma Aldrich

**From Barracuda Advanced Composites

The samples' manufacturing process can be described by the following steps:

1. Nanostructures non-covalent functionalization: in a beaker with $500 \mathrm{ml}$ of distilled water $1 \mathrm{~g}$ of carbon based nanostructure (CNT or MLG) were dispersed using a sonicator (Q500 from QSonica) at $42 \mathrm{KHz}$ with the appropriate amount of surfactant for 1 hour. The aqueous solution is later on dried in an oven at $100 \mathrm{C}$ for 24 hours;

2. Nanostructures dispersion: the appropriate amount of CNT or MLG (see Table 1) was dispersed into the epoxy resin using a high shear mixer (Turrax T-25 - IKA) at 17400 RPM for 1 hours followed by 1 hour sonication (Q500- QSonica);

3. Composite hand lay-up and post-cure: the nano-modified resin and hardener were mixed at $3: 1$ ratio in weight using a mechanical mixer (IKA RW-20) at 300 RPM for 5 minutes and degassed for another 5 minutes. The epoxy system was incorporated to carbon fibers using hand lay-up. The 8 layers composite plates (with thickness of $1.6 \mathrm{~mm}$ ) were cure on air for 24 hours followed by a post-cure in a hot press for 6 hours at $80 \mathrm{C}$ and $1.0 \mathrm{~atm}$ of pressure. The tensile samples followed the ASTM D-3039 standard $^{12}$. They were cut from the composite plates using a diamond saw.

The tensile tests were performed in an EMIC DL-1000 universal testing machine using a $100 \mathrm{KN}$ load cell and a $25 \mathrm{~mm}$ extensometer. All tests followed the ASTM D-3039 standard, which implies a velocity test of $2.0 \mathrm{~mm} / \mathrm{min}$. The infra-red spectroscopy analysis was performed using an Alpha Bruker FTIR spectrometer with an ATR-Ge from 4000 to $400 \mathrm{~cm}^{-1}$ at $2 \mathrm{~cm}^{-1}$ interval. Potassium bromide
$(\mathrm{KBr})$ and the non-covalent functionalized nanostructures were mixed in a ratio of $100 / 1$, then ground and pressed in a die to yield a transparent "window". FTIR samples were prepared under UV light in other to evaporate any water molecules. The Raman spectroscopy analysis was performed using a Horiba Jobin Yvon LABRAM-HR spectrometer with a helium-neon laser with $632.8 \mathrm{~nm}$ wavelength, with a CCD detector (charged coupled device) cooled by liquid nitrogen and an Olympus BHX microscope with lens of 10, 50 and 100X magnification. All Raman samples were in powder form. The atomic force analysis was performed using an MFP-3D-SAAtomic Force Microscopy from Asylum Research Incorporated. The samples for AFM analysis were prepared using a Leica UC6 ultra-microtome

\section{Data Analysis}

This investigation is based on multi-scale experimental analysis. At nano-scale FTIR and Raman spectroscopy study were performed. At sub-micron scale the nanostructures' morphology were described by atomic force microscopy. The macroscopic scale investigation was performed through tensile tests. The main objective is to create an integrated methodology that can correlate the three scales from atomistic to macroscopy analysis.

\subsection{FTIR and Raman Spectroscopy}

The first set of experiments were based on interactions between the carbon based nanostructures, i.e. CNT and MLG, and the surfactants. According to $\mathrm{Ju}$ et $\mathrm{al}^{13}$, the interaction between two substances (in our case, surfactant and carbon based nanostructures) can be identified by the presence of characteristic bands of the surfactant in the spectrum of functionalized nanostructures.

The FTIR spectra of the surfactants (SDBS and CO890) and their characteristic bands are shown in Figures $2 \mathrm{~A}$ and $2 \mathrm{~B}$, respectively. As described by Tao et $\mathrm{al}^{14}$, the FTIR characteristic peaks for SDBS are at $2922 \mathrm{~cm}^{-1}$ and $2853 \mathrm{~cm}^{-1}$ (relative to the axial deformations of $\mathrm{CH}$ of aliphatic groups). As the CO890 surfactant is a non-ionic one the characteristic peaks are different. Baomin et $\mathrm{al}^{15}$ defined three important peaks, i.e. the $2883 \mathrm{~cm}^{-1}$ band (axial deformation of $\mathrm{CH}$ in alkanes), the $947 \mathrm{~cm}^{-1}$ band (stretch CO) and the $841 \mathrm{~cm}^{-1}$ band (adjacent $2 \mathrm{H}$ in an aromatic ring). Interactions between carbon based nanostructures (CNT or MLG) and surfactants (SDBS or CO890) can be defined by the existence of such peaks into the FTIR spectrum.

Figures 2C-2D showed the FTIR spectra for CNT and MLG without functionalization, as grown, while figures $2 \mathrm{E}-2 \mathrm{~F}$ showed the FTIR spectra for CNT and MLG functionalized using SDBS. The SDBS' characteristic bands at $2922 \mathrm{~cm}^{-1}$ 


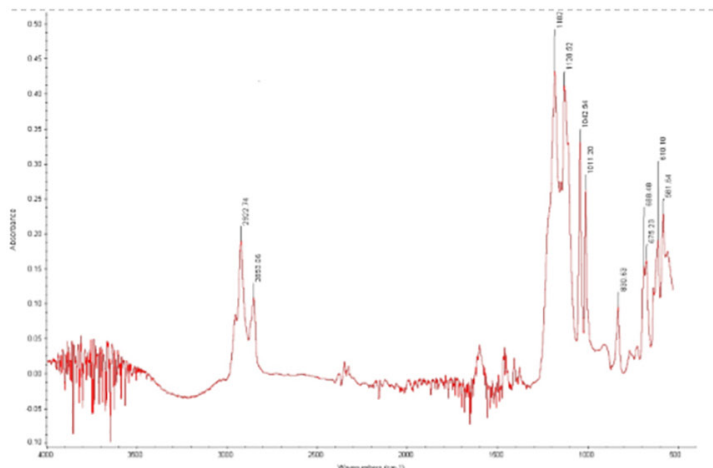

(a)

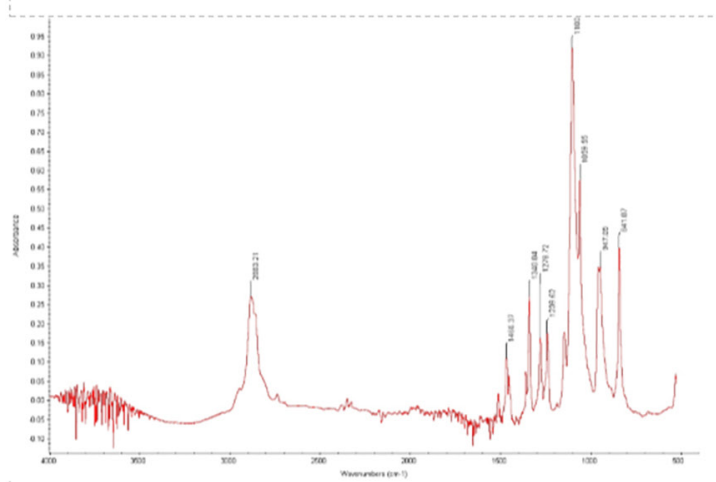

(b)

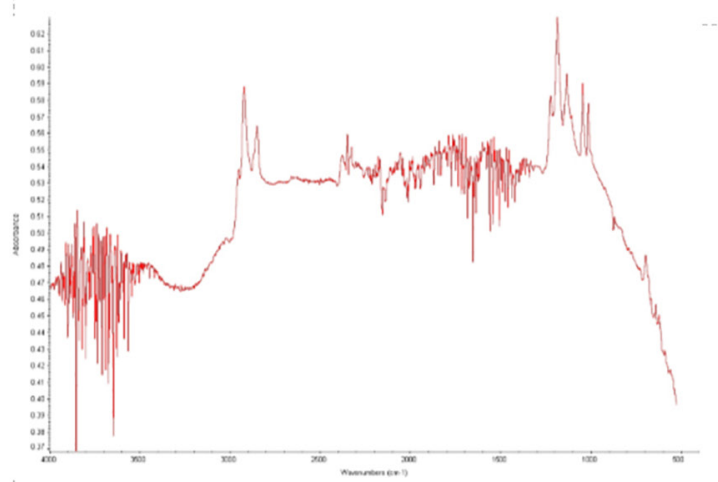

(c)

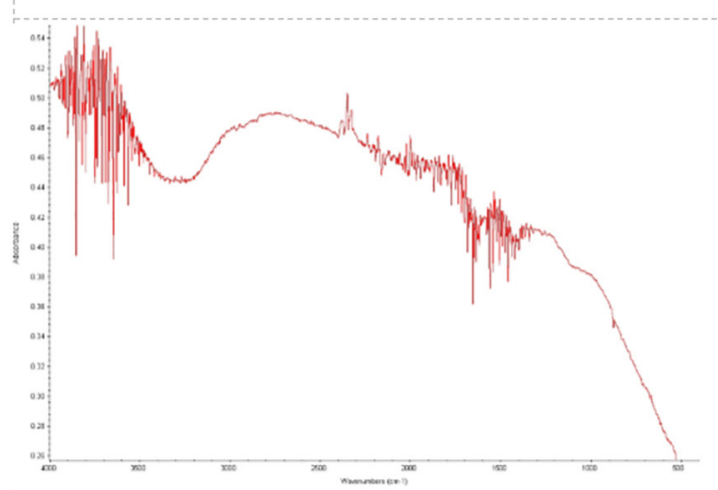

(d)

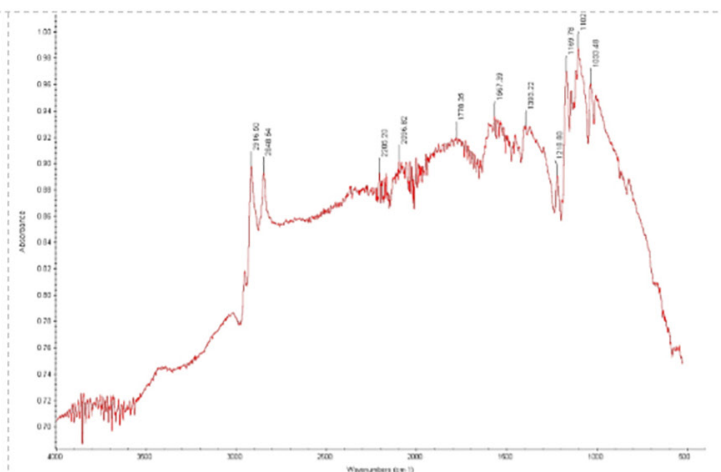

(e)

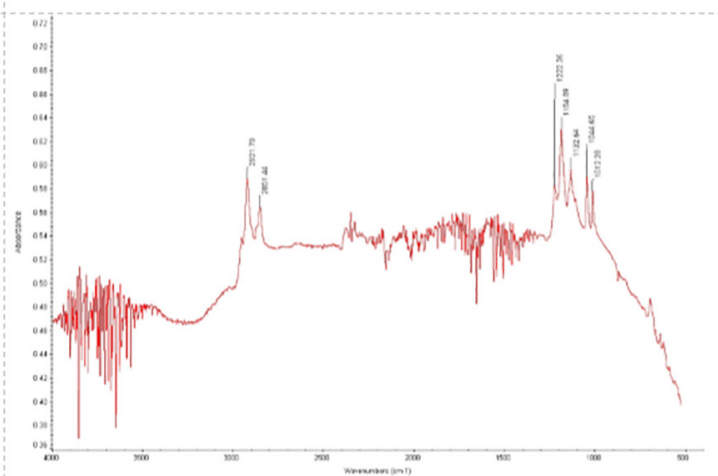

(f)

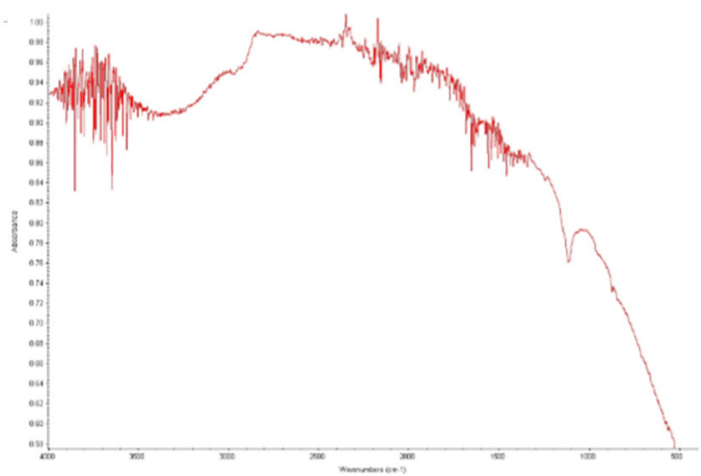

(g)

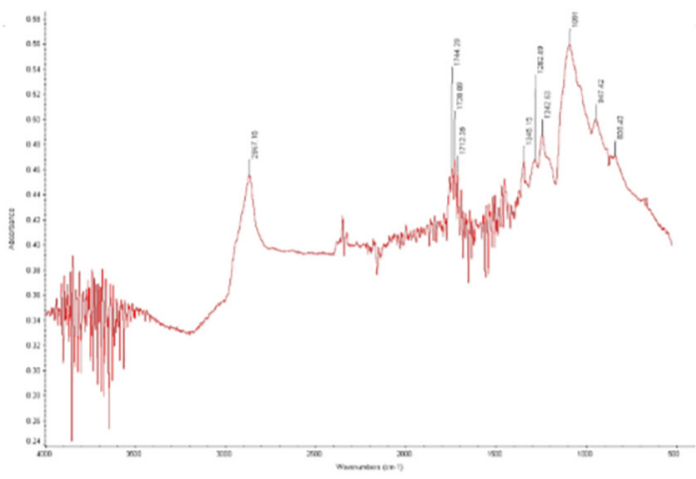

(h)

Figure 2. FTIR Spectra. (a) SDBS; (b) CO890; (c) CNT; (d) MLG; (e) SDBS+CNT; (f) SDBS+MLG; (g) CO890+CNT; (h) CO890+MLG; 


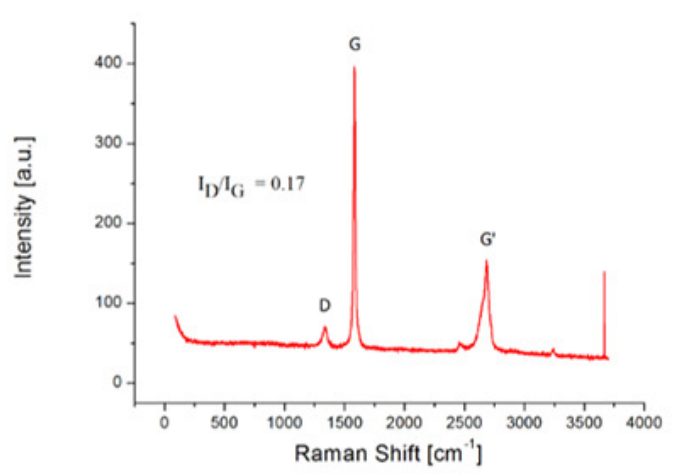

(a)

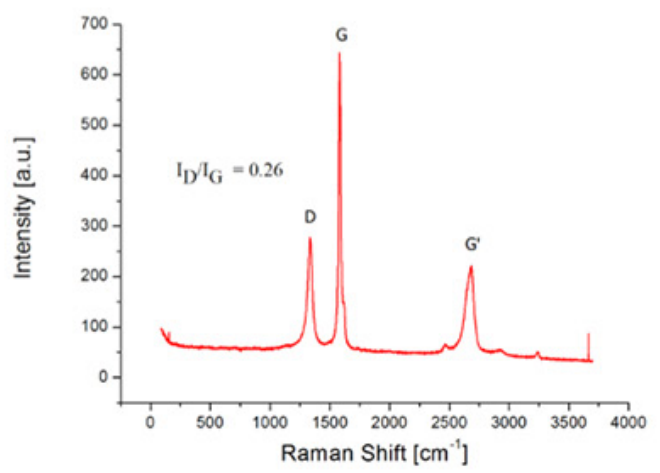

(b)

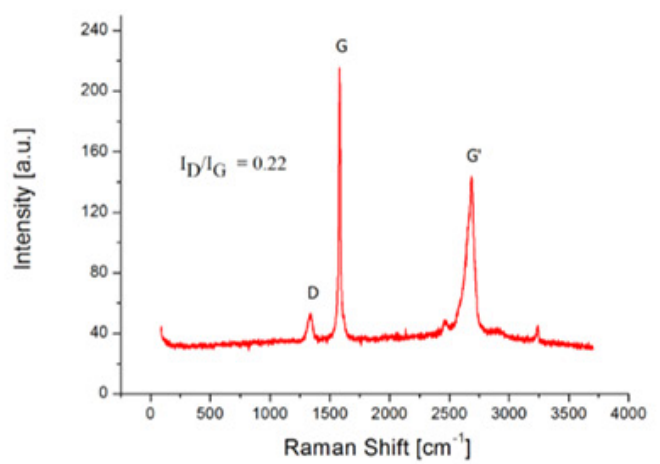

(c)

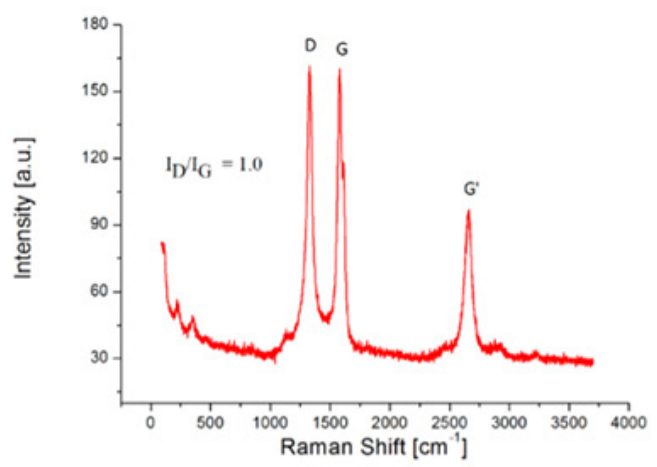

(d)

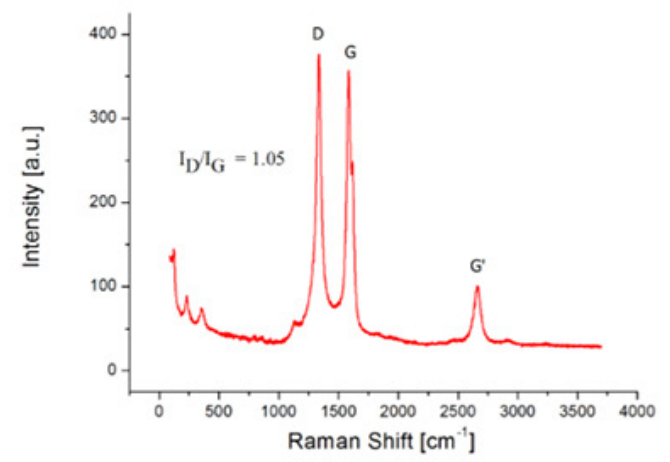

(e)

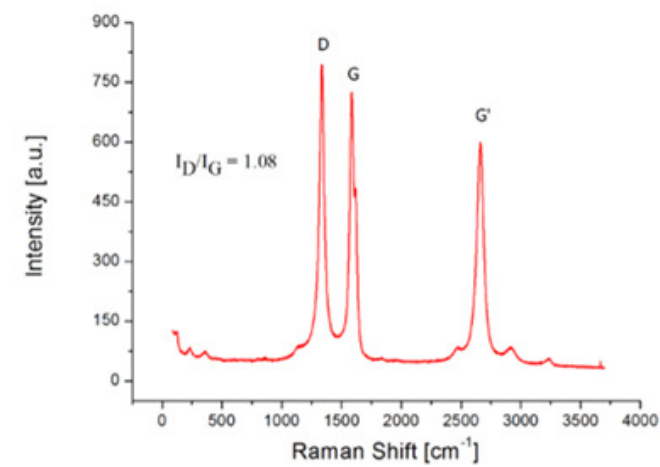

(f)

Figure 3. Raman Spectra. (a) MLG; (b) CO890+MLG; (c) SDBS+MLG; (d) CNT; (e) CO890+CNT; (f) SDBS+CNT; 
and $2853 \mathrm{~cm}^{-1}$ are identified for both cases. These bands are relative to the nonpolar portion of the surfactant and their presence in the functionalized nanostructured spectra indicates an interaction of this part of the molecule with the carbon nanostructures. It is also possible to observe that there was a decrease in the relative absorbance between these two bands when the nanostructures are mixed with the surfactant. According to Guo et $\mathrm{al}^{16}$, changes in band intensity suggest a strong interaction between substances after mixing. In the case of SDBS $+\mathrm{CNT}$ the decrease in relative absorbance was higher than in SDBS+MLG. These data indicate that both MLG and CNT interacted with SDBS and that the affinity of this surfactant is higher with CNT than with MLG.

When the CO890 interaction was analyzed, a different scenario was observed. The CO890+CNT seem not have

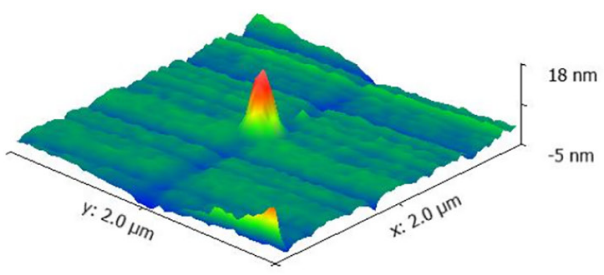

(a)

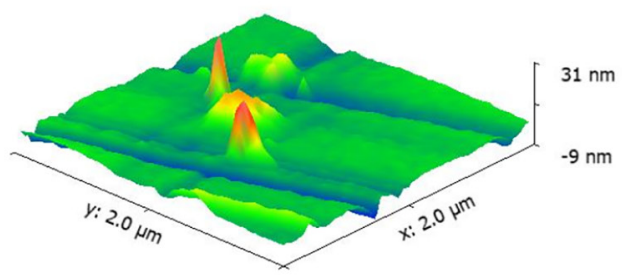

(b)

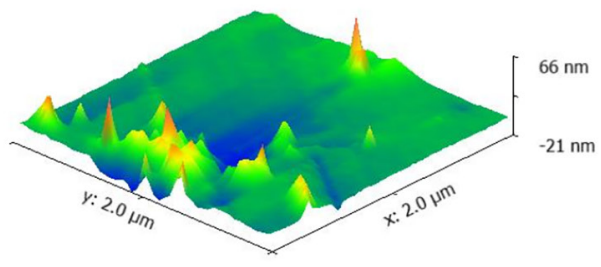

(c)

Figure 4. AFM Observations. (a) 0.075 wt. $\%$ CNT; (b) 0.15 wt.\% CNT; (c) 0.30 wt.\% CNT; any interaction (Figure 2G), as no CO890 characteristic peaks were identified into the functionalized specimen. For the CO890+MLG specimen, the CO890 three characteristic bands were observed, i.e. $2883 \mathrm{~cm}^{-1}, 947 \mathrm{~cm}^{-1}, 841 \mathrm{~cm}^{-1}$ (see Figure 2H). This result indicates that there is an interaction of the apolar part of this surfactant with the MLG nanostructure. A complementary analysis can be done using Raman spectroscopy ${ }^{17}$.

In the Raman spectra for CNT and MLG, it is possible to observe the bands characteristic to the $\mathrm{sp}^{2}$ hybridized carbon structures. The band $\mathrm{G}$ is located at approximately $1580 \mathrm{~cm}^{-1}$ corresponds to the stretch of the $\mathrm{C}-\mathrm{C}$ bond and the $\mathrm{G}^{\prime}$ band (also called 2D) can be identified at approximately 2725 $\mathrm{cm}^{-1}$. Note that according to Kim et al. ${ }^{18}$, in nano-crystalline

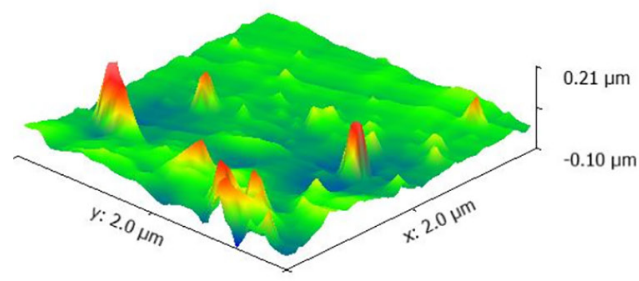

(a)

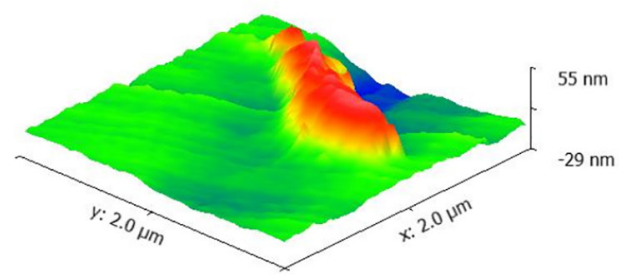

(b)

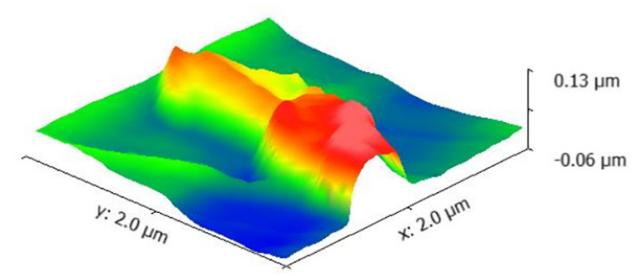

(c)

Figure 5. AFM Observations. (a) 0.075 wt.\% MLG; (b) 0.15 wt.\% MLG; (c) 0.30 wt.\% MLG; 
Table 2. Tensile tests summary

\begin{tabular}{ccccc}
\hline Group ID & Ultimate Strength $[\mathrm{MPa}]$ & Strain at Failure $[\mu \varepsilon]$ & Stiffness $[\mathrm{GPa}]$ & Toughness $\left[\mathrm{MJ} / \mathrm{m}^{3}\right]$ \\
\hline Control & $575.76 \pm 29.38$ & $55.44 \pm 5.20$ & $12.98 \pm 0.67$ & $14.21 \pm 2.40$ \\
CNT-0.075 & $666.17 \pm 27.95$ & $70.10 \pm 7.54$ & $10.93 \pm 0.35$ & $23.96 \pm 4.26$ \\
CNT-0.150 & $680.86 \pm 36.15$ & $63.73 \pm 2.79$ & $11.91 \pm 0.39$ & $20.96 \pm 1.27$ \\
CNT-0.300 & $630.65 \pm 64.89$ & $57.04 \pm 3.52$ & $12.94 \pm 0.72$ & $17.68 \pm 2.27$ \\
MLG-0.075 & $666.32 \pm 20.69$ & $56.76 \pm 1.69$ & $13.30 \pm 0.42$ & $17.34 \pm 0.92$ \\
MLG-0.150 & $649.43 \pm 48.73$ & $54.79 \pm 3.01$ & $12.97 \pm 1.72$ & $16.65 \pm 0.27$ \\
MLG-0.300 & $580.35 \pm 25.05$ & $51.04 \pm 3.13$ & $13.88 \pm 0.37$ & $13.72 \pm 1.69$ \\
\hline
\end{tabular}

samples or with imperfections in the structure, it is possible to observe the appearance of the D band at approximately $1350 \mathrm{~cm}^{-1}$. Englert et al. ${ }^{17}$ observed that an increase in the ID/IG ratio may be related to a satisfactory functionalization of the carbon nanostructures. The ID/IG ratios shown in Figure 2 are the mean of the values obtained in the different analyzes for each sample. For the MLG cases, the ID/IG ratio without surfactant was equal to 0.17 (Figure 3A). This ratio, however, changed to 0.26 (Figure $3 \mathrm{~B}$ ) and 0.22 (Figure 3C) when the MLG were functionalized with CO890 and SDBS, respectively. It is possible to conclude that for MLG, CO890 provided a better functionalization. It can be seen in the CNT cases, the ID/IG ratio of 1.0 (Figure 3D) changed to 1.05 with CO890 functionalization (Figure 3E) and to 1.08 with SDBS (Figure 3F). These values indicate that the functionalization of CNT is more effective using SDBS than CO890, a conclusion similar to that obtained in FTIR analysis.

As it can be observed by FTIR and Raman spectroscopy data each carbon based nanostructure has more affinity to individual surfactant. The best options are MLG+CO890 and $\mathrm{CNT}+\mathrm{SDBS}$. The interaction between surfactantcarbon based nanostructures is an evidence of effective functionalization. A good functionalization can be translated into strong bonds at the fiber/matrix interface, in which can have direct influence into the overall mechanical properties. However, as the carbon based nanostructure concentrations were different, it is important to establish a relation between these concentrations and the nanostructure morphology formed during the dispersion process. Lee et al. ${ }^{19}$ pointed out that these sub-micron nanostructures can affect the overall composite mechanical properties. Moreover, Shah and Batra ${ }^{20}$ went further, as they pointed that non-covalent functionalization has direct effect into nanostructure morphology. This analysis must be performed using atomic force microscopy at sub-micron scale.

\subsection{Atomic Force Microscopy Analysis}

CNT based nano-structures are mainly unidirectional one in a "spike-like" shape. As concentrations increased not only the spatial distribution was uniformly wide spread, but an increase on height was also observed (Figure 4A-C). This phenomenon can be attributed to the usage of surfactants (SDBS). SDBS inhibits cluster/agglomeration formation but at same time "facilitates" chemical bonds between small quantities of CNT can create Van der Waals bonds between them and the SDBS.

MLG nanostructures have a "wall-like" shape. This shape was expected as we are dealing with multi-layer graphene nanostructures. Again, the increase on concentration of CO890-MLG into the epoxy system lead to increase on nanostructure height and width. CO890 proven to be effective into prevent cluster/agglomeration formation. Moreover, like SDBS some Van der Waals bonds can be formed between MLG nanostructures. This hypothesis can be confirmed by the increase on MLG nanostructure size (Figure 5A-C).

Large nanostructures such as the ones observed in Figure 4C can have an opposite effect into nano-reinforcement. As it can be seen as "hot-spots" for stress concentration formation. Small and wide spread nano-size structures (see Figure 4A-5A) can act in favor of blocking and deflecting crack propagation at nano/micro-level.

\subsection{Tensile Tests Analysis}

Based on ANOVA - analysis of variance of tensile test stiffness data, it is possible to conclude that there is no significant difference on mean values at level of significance of 0.05 (see Table II). Therefore, it is likely to infer that nano-structures addition to epoxy system did not affect the overall composite Young's moduli. Small variations can be attributed to different factors, e.g. local in-homogeneity, geometric imperfections or localized stress concentrations. The ultimate stresses, however, seem to be largely influenced by the nanostructures' addition to the epoxy system. The mean values for CNT-0075, CNT-015 and MLG0075 groups are statistically different from the control group. It is a wellknown fact that ultimate stress is related to crack formation and its propagation during the tensile test. The good results from CNT-0075, CNT-015 and MLG-0075 groups can be due to nanostructures' effective dispersion into the matrix. These nanostructures, shown in Figure 4A-4B-5A, may act as barriers by blocking and/or deflecting crack propagation, which lead to increase on energy dissipation. The average increase on ultimate stress was around $18.25 \%$. 
Small concentrations $(0.075 \mathrm{wt} . \%$ and $0.15 \mathrm{wt} . \%)$ seem to act in favor of a more uniform distribution, which makes the crack propagation most likely to be blocked or deflected in a more effective away. This more efficient dispersion process can be attributed to the sonication process. During sonication vibration is induced to the nanostructures (CNT or MLG) up to a point, when the fundamental frequency of CNT or MLG is reached. At this moment, the resonance effect takes place and, CNT or MLG are uniformed spread. For larger concentrations $(0.15 \mathrm{wt} . \%$ and $0.30 \mathrm{wt}$. $\%$ for MLG and 0.30 wt. \% for CNT), the resonance effect is reduced due to the nanostructures interactions, as they "bump to each other".

Another important issue is the composites' toughness, which is represented by the area below the stress-strain curve. An ANOVA analysis detected that all mean values are statistically different, with level of significance of 0.05 , from the control sample group. The average increase on toughness with respect to control samples group was around $68.61 \%$. A possible explanation for such increase relays in two factors: the first one is a uniform or at least quasi-uniform distribution of nano-size structures. Notice that CNT and MLG nanostructures have an elastic moduli of $1000 \mathrm{GPa}$. These nano-size "barriers" make crack formation and propagation more difficult. The second factor is the possible increase on fiber/matrix adhesion due to chemical bonds formed by the usage of surfactants (SDBS or CO890). This observation was also corroborated by Shah et al ${ }^{20}$, which stated that fiber/matrix interaction is also improved by the surfactant usage. The increase on failure strain (see Table II) is also an indication that nano-modified composites can sustain more damage previously to failure.

The size (around $180 \mathrm{~nm}$ ) and spatial distribution of MLG-030 nanostructure (see Figure 5C) formed could be the reason for small decrease on toughness with respect to control samples group. In all other cases, the nanostructures were able to provide an increase on toughness moduli. Furthermore, if only toughness is considered small concentrations lead to more effective damage tolerance and more effective energy dissipation.

In sum, CNT and MLG nanostructures are viable options for epoxy matrix nano-reinforcement. The usage of surfactants is highly recommended, SDBS for CNT and CO890 for MLG. The nanostructure size effect is also an important issue when tensile tests are considered. A rule of thumb can be smaller nanostructures can lead to better results with respect to strength and toughness. Finally, stiffness seems to be not affected by the carbon based nano-reinforcement, while strength and toughness are greatly influenced by CNT/MLG.

\section{Conclusions}

A multi-scale analysis of carbon/epoxy nano-modified composites by carbon nanotubes or multi-layer graphene was performed. FTIR analysis and Raman spectroscopy indicated a strong chemical interaction between CO890 and MLG and from SDBS and CNT. These interactions, mainly by the Van der Waals bonds, prevent the cluster/agglomeration formation when these nano-size structures are dispersed into epoxy systems and incorporated to carbon fibers. AFM observations detected "spike-like" nanostructures when CNT are dispersed into epoxy systems, while "wall-like" nano and sub-micro size structures were formed by CO890-MLG were dispersed into epoxy systems. No significant changes on stiffness were observed with CNT or MLG addition. However, strength and toughness were greatly influenced by the nanostructure type (CNT or MLG), size and spatial distribution. The average increase on strength was around $18 \%$, while toughness had an increase on average of approximately $62 \%$. SDBS-CNT and CO890-MLG at small concentrations seem to be best options for carbon/epoxy composites nano-reinforcement.

\section{Acknowledgements}

The authors would like to acknowledge the financial support provided by the Brazilian Research Council (CNPq) grant 304646/2014-8 the Air Force Office of Scientific Research (AFOSR) grant FA9550-14-1-0377. The authors are also grateful to the UFMG's Center of Microscopy and Microanalysis for the technical support and the Nacional Grafite Incorporated for supplying the graphite from which the graphene blocks were obtained.

\section{References}

1. Zhu J, Chen M, He Q, Shao L, Wei S, Guo Z. An overview of the engineered graphene nanostructures and nanocomposites. RSC Advances. 2013;3(45):22790-22824.

2. Kuilla T, Bhadra S, Yao D, Kim NH, Bose S, Lee JH. Recent advances in graphene based polymer composites. Progress in Polymer Science. 2010;35(11):1350-1375.

3. Lee C, Wei X, Kysar JW, Hone J. Measurements of the elastic properties and intrinsic strength of monolayer graphene. Science. 2008;321(5887):385-388.

4. Ávila AF, Oliveira AM, Munhoz VC, Monteiro EC, Pereira GC. Graphene-Carbon Nanotubes Hybrids for Composite Materials. In: Proceedings of the $56^{\text {th }}$ AIAA/ASME/ASCE/ AHS/ASC Structures, Structural Dynamics, and Materials Conference; 2015 Jan 8-12; Kissimminie, FL, USA. p. 1-12.

5. Kim M, Park YB, Okoli OI, Zhang C. Processing, characterization, and modeling of carbon nanotube-reinforced multiscale composites. Composites Science and Technology. 2009;69(3/4):335-342.

6. Tkalya EE, Ghislandi M, de With G, Koning CE. The use of surfactants for dispersing carbon nanotubes and graphene to make conductive nanocomposites. Current Opinion in Colloid \& Interface Science. 2012;17(4):225-232.

7. Pu NW, Wang CA, Liu YM, Sung Y, Wang DS, Ger MD. Dispersion of graphene in aqueous solutions with different types of surfactants and the production of graphene films by spray or drop coating. Journal of the Taiwan Institute of Chemical Engineers. 2012;43(1):140-146. 
8. Mittal V. Functional polymer nanocomposites with graphene: A review. Macromolecular Materials and Engineering. 2014;299(8):906-931.

9. Bystrzejewski M, Huczko A, Lange H, Gemming T, Büchner B, Rümmeli MH. Dispersion and diameter separation of multiwall carbon nanotubes in aqueous solutions. Journal of Colloid and Interface Science. 2010;345(2):138-142.

10. Lacerda RG, Teo KBK, The AS, Yang MH, Dalal SH, Jefferson DA, et al. Thin-film metal catalyst for production of multi-wall and single-wall carbon nanotubes. Journal of Applied Physics. 2004;96(8):4456-4462.

11. Avila AF, Munhoz VC, Menezes NC, Da Silva CF, Leão SG. Non-covalent functionalisation of carbon-based nanostructures and its application to carbon/epoxy composites. International Journal of Nanotechnology. 2016;13(8/9):573-583.

12. ASTM International. ASTM D3039/D3039M - Standard Test Method for Tensile Properties of Polymer Matrix Composite Materials. West Conshohocken: ASTM International; 2017.

13. Ju L, Zhang W, Wang X, Hu J, Zhang Y. Aggregation kinetics of SDBS-dispersed carbon nanotubes in different aqueous suspensions. Colloids and Surfaces A: Physicochemical and Engineering Aspects. 2012;409:159-166.
14. Tao H, Zhang Y, Gao Y, Sun Z, Yan C, Texter J. Scalable exfoliation and dispersion of two-dimensional materials - an update. Physical Chemistry Chemical Physics. 2017;19(2):921960.

15. Baomin W, Ruishang J, Ruying Z. Dispersion of graphene nanoplatelets in aqueous solution. Journal of Nanoscience and Nanotechnology. 2017;17(12):9020-9026.

16. Guo Z, Wang S, Wang G, Niu Z, Yang J, Wu W. Effect of oxidation debris on spectroscopic and macroscopic properties of graphene oxide. Carbon. 2014;76:203-211.

17. Englert, JM, Vecera P, Knirsch KC, Shafer RA, Hauke F, Hirsch A. Scanning-Raman-Microscopy for the statistical analysis of covalently functionalized graphene. ASC Nano. 2013;7(6):54725482 .

18. Kim YA, Fujisawa K, Muramatsu H, Hayashi H, Endo M, Fujimori T, et al. Raman Spectroscopy of boron-doped singlelayer graphene. ASC Nano. 2012;6(5):6293-6300.

19. Lee SH, Lee DH, Lee WJ, Kim SO. Taylored assembly of carbon nanotubes and graphene. Advanced Functional Materials. 2011;21(8):1338-1354.

20. Shah PH, Batra RC. Effect of Covalent Functionalization on Young's Modulus of a Single-Wall Carbon Nanotube. In: Tserpes KI, Silvestre N, eds. Modelling of Carbon Nanotubes, Graphene and their Composites. Berlin: Springer Verlag; 2014. p. 111-134. 